\title{
Comparison of sound localization performance between virtual and real three-dimensional immersive sound field
}

\author{
Dae-Gee Kang*, Yukio Iwaya, Ryota Miyauchi and Yôiti Suzuki \\ Research Institute of Electrical Communication and Graduate School of Information Sciences, Tohoku University, \\ 2-1-1 Katahira, Aoba-ku, Sendai, 980-8577 Japan
}

(Received 19 August 2008, Accepted for publication 21 November 2008)

Keywords: Head-related transfer functions (HRTFs), Virtual auditory display, Sound localization PACS number: 43.66.Qp [doi:10.1250/ast.30.216]

\section{Introduction}

There is an increasing demand to realize a high sense of presence in communication systems and home entertainment systems. As a consequence, acoustic techniques that synthesize/reproduce information about the accurate location of sound sources in three-dimensional auditory spaces have become crucially important. A system which renders threedimensional auditory spaces is termed a virtual auditory display (VAD) [1]. A conventional but effective acoustic technique to realize VADs is the synthesis of head-related transfer functions (HRTFs) [2]. In this method, sound localization in three-dimensional auditory space is rendered by convolving head-related impulse responses (HRIRs), which are the inverse FFT of HRTFs, related to locations of sound sources. To realize high accuracy of sound localization, a key is to synthesize HRTFs according to the listener's movement, and thus several systems have been proposed which are responsive to a listener's head movement [3-5]. However, there have been very few proposals of VADs that are responsive to a listener's translational movement and which would enable a listener to move freely in a three-dimensional space.

In this study, a VAD which enables a listener to move freely in a small room three-dimensionally was constructed. Then its performance was evaluated by comparing distance errors of a sound source between a real three-dimensional sound field and a virtual one synthesized by the VAD.

\section{VAD system}

The VAD system was installed in a soundproof room $(4.7 \mathrm{~m}(\mathrm{~W}) \times 2.8 \mathrm{~m}(\mathrm{D}) \times 3.0 \mathrm{~m}(\mathrm{H}))$. The system consisted of a personal computer $(3.6 \mathrm{GHz}$ Pentium $4 \mathrm{CPU}, 2$ GByte memory), a sound presentation device, and a three-dimensional ultrasound sensor system (IS-900; InterSense) as shown in Fig. 1. Linux (Kernel 2.6) was used as the operating system.

A LADOMi (Localization Auditory Display with Opened ear-canal for Mixed Reality) [6] was employed as the sound presentation device. For the LADOMi, rather than employing earphones, two flat-panel loudspeakers (Fig. 2(a), FPS0204M7, FPS Co. Ltd.) were attached to a helmet as shown in Fig. 2(b). Therefore, both ear canals were exposed to the air; acoustic impedance near the external ear canal

*e-mail: dkrehd@ais.riec.tohoku.ac.jp change little. According to Møller [7], if the reproduction device is a Free air Equivalent Coupling to the ear (FEC) headphone that remains unchanged, then for acoustic impedance near the external ear canal, sound localization becomes accurate. In fact, LADOMi has a good FEC headphone characteristic. For that reason, it is more accurate than the earphone/headphone characteristic in terms of sound localization. This device is classified as a transaural reproduction system, which is similar to so-called near-phones [8].

For the ultrasonic position sensor (InterSense IS-900), six sonic strip bars which generate ultrasonic waves were installed on the ceiling at equal intervals to cover the whole room. A tracker was attached at the center of the top of the LADOMi to detect movement information of the listener $(x, y$, $z$, yaw, roll, pitch). The processing flow in our VAD system is as shown in Fig. 1. First, the system obtains information on the relative position of a sound source to be rendered by using the relationship between the presented sound source and the head direction and position of the listener calculated by the sensor system in the three-dimensional space. Second, to realize smooth rendering of the sound source three-dimensionally, the system calculates a suitable HRIR for each ear by interpolating four HRIRs near the position to be localized in the HRIR data set of the system based on the method described in our previous paper [9]. Finally, a virtual sound image is generated by convolving the two interpolated HRIRs for right and left ears of the listener with a sound source, and the results are then outputted via LADOMi. The HRTFs used in the experiment were measured for each listener with loudspeakers constantly $1.5 \mathrm{~m}$ away from the center of the listener's head in an anechoic room. To express the distance between the sound source to a listener the amplitude of the sound signal was controlled by the inverse-square law; i.e., the sound pressure level for the virtual sound source was decreased by $6 \mathrm{~dB}$ to double the distance between the sound source and the listener. The sound amplitude was kept constant to prevent divergence when the distance became less than $20 \mathrm{~cm}$. The length of the digital filter for synthesis of HRTFs was set at 512 points and the sampling frequency was $48 \mathrm{kHz}$.

\section{Experiment}

The performance of the constructed VAD system was examined using a listening experiment under two environments, i.e., a virtual sound field (Fig. 3(a)) and a real one 


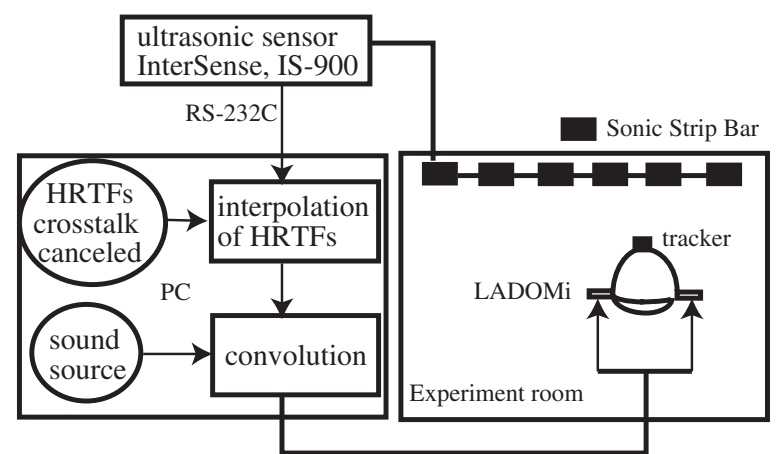

Fig. 1 System configuration and processing flow.

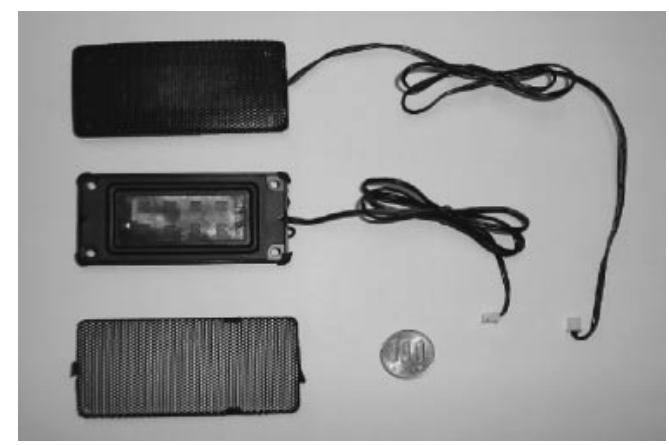

(a) FPS loudspeaker

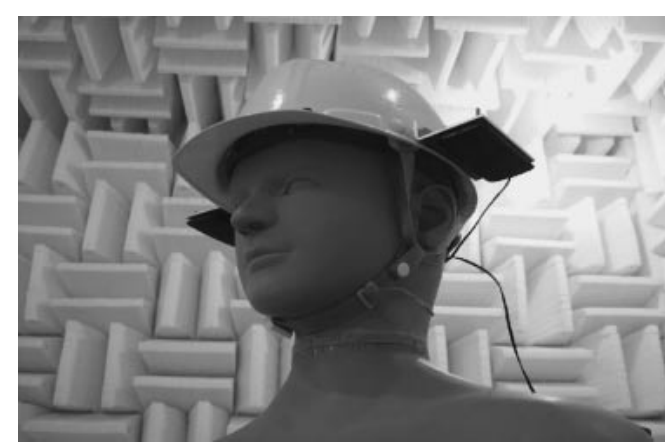

(b) LADOMi

Fig. 2 Virtual auditory display system (LADOMi).

(Fig. 3(b)). In the virtual sound field, a virtual sound source constructed by the VAD system was presented. On the other hand, in the real sound field, a sound source was presented using a small omni-directional loudspeaker which consisted of 32 electrodynamic loudspeakers and had a diameter of $7.5 \mathrm{~cm}$ (Fig. 3(c)). As previously mentioned, the experiment was performed in a soundproof room $(4.7 \mathrm{~m}(\mathrm{~W}) \times 2.8 \mathrm{~m}(\mathrm{D}) \times$ $2.3 \mathrm{~m}(\mathrm{H})$ ). Listeners were four young adults (two males and two females) with normal hearing acuity. The positions of the four presented sound source are shown in Table 1, and Fig. 4. In the $x-y$ plane, the positions of the four presented sound sources were arranged at the four corners in the room as dispersedly as possible. On the $z$-axis, the positions of the four presented sound sources were set up at $190 \mathrm{~cm}$ or more from the floor to avoid bumping against the loudspeaker while the listener was experimenting. All listeners were blindfolded so that they could not see the positions of loudspeakers, and were

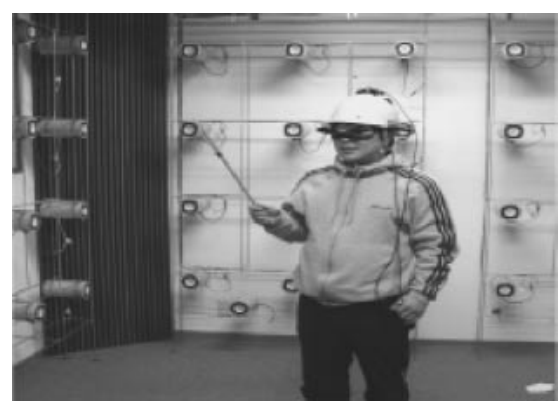

(a) Virtual experiment

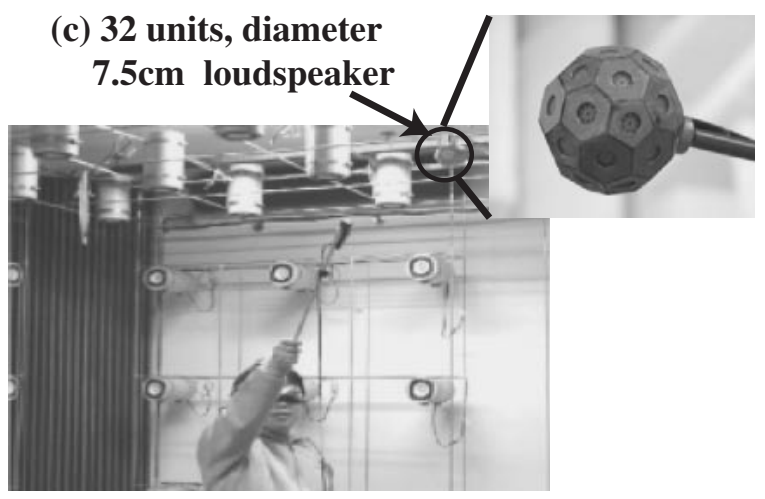

(b) Real experiment

Fig. 3 Experiment overview.

Table 1 Position of the presented sound source.

\begin{tabular}{cccc}
\hline Position & $x[\mathrm{~cm}]$ & $y[\mathrm{~cm}]$ & $z[\mathrm{~cm}]$ \\
\hline P1 & 320 & 140 & 205 \\
P2 & 315 & 40 & 190 \\
P3 & 130 & 55 & 200 \\
P4 & 130 & 155 & 210 \\
\hline
\end{tabular}

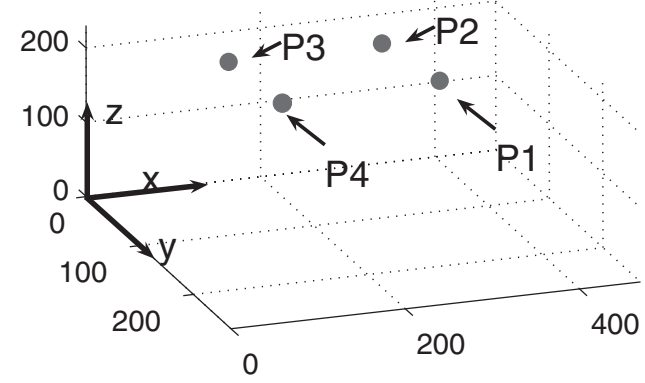

Fig. 4 The positions of the presented sound source from P1 to P4.

allowed to attempt localizing a presented sound source only one time for each position. A 120-second-long piece of classical music was used as the sound source. Within that interval, the listener was asked to seek the sound source under conditions of free movement around the room. Listeners indicated the perceived position using a long stick with a position tracker at its tip. The coordinate information when listeners judged the sound source position was obtained by 


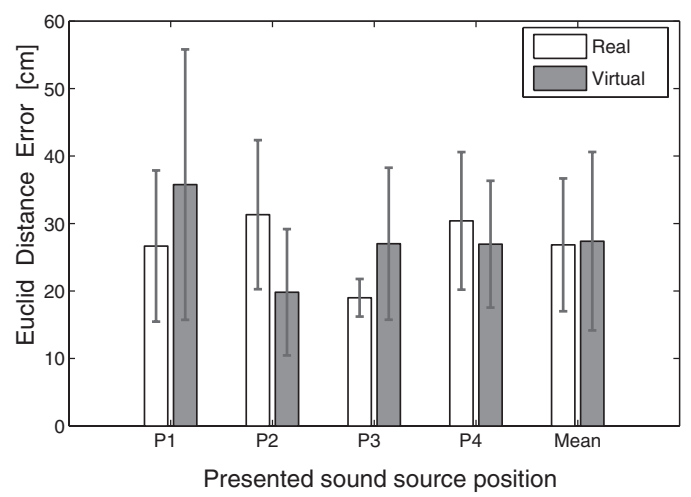

Fig. 5 Euclidean distance errors for each presented sound source position of real and virtual environments.

a position tracker. In the experiment with a virtual sound source, the virtual sound source was generated with the listener's own HRTFs since that he/her own set of HRTFs is more accurate for sound localization than other HRTFs [10]. The HRTFs for elevation below -40 degrees were replaced by those at -40 degrees because of an accuracy problem of HRTFs below -40 degrees.

\section{Results and discussion}

Euclidean distance errors from the presented sound source position to the perceived sound source position were calculated for each environment and each presented sound source position with respect to each listener. Note that direction errors were not available for evaluating the performance of this VAD. The listeners were able to move freely in the experimental room. Consequently, the distances between the sound source and the listener differed among listeners, although the position of the sound source was identical. The absolute direction, therefore, is beyond its systematic control. We specifically examined the difference of the distance errors between the real and the virtual sound sources to evaluate this VAD. The mean values of these distance errors are shown in Fig. 5. The vertical bars represent the standard deviations. The mean values of distance errors of all positions were 26.8 and $27.4 \mathrm{~cm}$ for the real and virtual environments, respectively. These results showed that the accuracy of sound localization in three-dimensional space was approximately the same between the real and the virtual environments. This observation was confirmed in a 2 (real and virtual environments) $\times 4$ (positions of the presented sound source) repeated measurements analysis of variance (ANOVA), which showed no significant main effect and interaction.

To investigate the difference between the real and virtual environments, the three-dimensional coordinate was divided into two components, the $x-y$ plane being regarded as the horizontal component and $z$-axis being regarded as the vertical component. Euclidean distance errors were calculated for each component and each environment with respect to each listener and each position, the results being shown in Fig. 6. A discrepancy in the tendency of the misalignment between the real and the virtual environments was observed. The distance error of the virtual environment was small compared with that of the real one in the horizontal

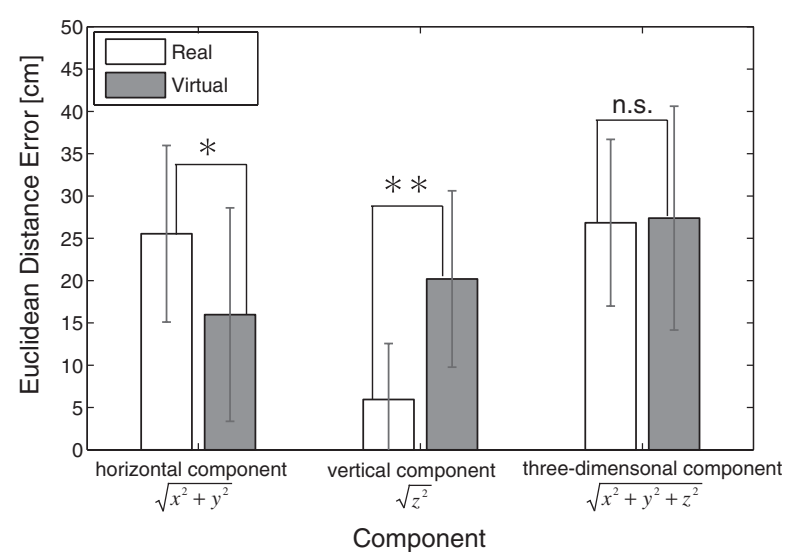

Fig. 6 Euclidean distance error for each component between real and virtual environments.

component. In contrast, the distance error in the vertical component was opposite that of the horizontal component. To verify this result, we conducted a $t$-test with regard to the distance error of each component between the real and virtual environments. As a result, a significant difference was obtained for the horizontal component $(t[16]=2.219, p<$ 0.05 : two sided $)$ and the vertical component $(t[16]=-4.960$, $p<0.01$ : two sided) between the real and virtual environments. This result revealed that the virtual environment was more accurate than the real one in the horizontal component, while the real environment was more accurate than the virtual one in the vertical component.

In case of the real environment, distance error of the horizontal component was larger than that of the vertical component. One possible reason for this result may be the effect of reverberation in a room. According to the accuracy of the sound localization with and without reverberation examined by Shinn-Cunningham [11], reverberation degrades perception of the sound source direction, but enhances distance perception. In our experiment, the reverberation existed in the real environment because the experiment room had a reverberation time of almost 0.2 seconds, whereas the reverberation time was 0 seconds in the virtual environment. Thus the sound source direction might be blurred with the reverberation, resulting in larger distance error in the horizontal component, and the vertical component, which can be considered to be related to distance perception, might be enhanced by reverberation, resulting in smaller distance error.

\section{Conclusion}

In this study, a new type of VAD, which allows a listener to freely move in a room, different from ordinary VAD was constructed and its performance was evaluated by means of a sound searching experiment with a real sound source and a virtual sound source generated by the VAD. No noticeable difference was observed between the real and the virtual environment in terms of total distance error. However, the horizontal and vertical components of the distance errors between real and virtual environments exhibited different tendencies. We specifically examined distance errors only to evaluate the performance of this VAD. For that reason, it 
is difficult to compare this VAD with ordinary VAD for performance and to note the effect of a listener's free movement in this experiment. Therefore, it is left as a subject for our future work.

\section{Acknowledgment}

This study was partly supported by the GCOE program of the Graduate School of Technology, Tohoku University.

\section{References}

[1] E. M. Wenzel, F. L. Wightman and S. H. Foster, "A virtual display system for conveying three-dimensional acoustic information," in 32nd Annu. Meet. Hum. Fact. Ergon. Soc., Human Factors and Ergonomics Society, Santa Monica (1988).

[2] F. L. Wightman and D. J. Kistler, "Headphone simulation of free-field listening. II: Psychophysical validation," J. Acoust. Soc. Am., 85, 868-878 (1989).

[3] J. Kawaura, Y. Suzuki, F. Asano and T. Sone, "Sound localization in headphone reproduction by simulating transfer functions from the sound source to the external ear," J. Acoust. Soc. Jpn. (J), 45, pp. 756-766 (1989), English translation appears in: J. Acoust. Soc. Jpn. (E), 12, 203-216, (1991).
[4] D. Miller and E. M. Wenzel, "Recent developments in SLAB: A software-based system for interactive spatial sound synthesis," Proc. 2002 Int. Conf. Auditory Display (2002).

[5] S. Yairi, Y. Iwaya and Y. Suzuki, "Development of virtual auditory display software responsive to head movement," Trans. Virtual Reality Soc. Jpn., 11, 437-446 (2006).

[6] R. Shibuya, Y. Ishida, Y. Iwaya, M. Sakata and Y. Suzuki, "Development of auditory display system with flat panel speaker for mixed reality," 3rd Information Science and Technology Forum, FIT2004, pp. 285-286 (2004) (in Japanese).

[7] H. Møller, "Fundamentals of binaural technology," Appl. Acoust., 36, 171-218 (1992).

[8] D. H. Cooper and J. L. Bauck, "Prospects for transaural recording," J. Audio Eng. Soc., 37(1/2), pp. 3-19 (1989).

[9] K. Watanabe, S. Takane and Y. Suzuki, "A novel interpolation method of HRTFs based on the common-acoustical-pole and zero model," Acustica - Acta Acustica, 91, 958-966 (2005).

[10] M. Morimoto and Y. Ando, "On the simulation of sound localization,” J. Acoust. Soc. Jpn. (E), 1, 167-174 (1980).

[11] B. Shinn-Cunningham, "Learning reverberation: Considerations for spatial auditory displays," Proc. 2000 Int. Conf. Auditory Display, pp. 126-133 (2000). 